\title{
Acessibilidade estrutural na cidade de São Carlos, SP, Brasil
}

\author{
Denise Capasso da Silva ${ }^{1}$ e Antônio Nélson Rodrigues da Silva ${ }^{2}$
}

\begin{abstract}
Resumo: O Mapa de Acessibilidade Estrutural é a representação geográfica comparativa dos níveis de acessibilidade por modo de transporte para diferentes tipos de oportunidades de geração de viagens. A ferramenta, que é baseada na integração das considerações a respeito do sistema de transporte e do uso do solo, foi validada em uma aplicação na cidade do Porto, Portugal. A proposta do presente trabalho é utilizar o método em questão para análise dos níveis de acessibilidade da cidade de São Carlos. Para isso foram utilizados dados censitários e resultados de uma pesquisa Origem e Destino. Assim, esse trabalho mostra que o Mapa de Acessibilidade Estrutural é um recurso que auxilia a tomada de decisão quando do planejamento do transporte urbano, como pôde ser observado no estudo de caso feito para a cidade São Carlos. Ainda, a aplicação mostra a viabilidade da utilização do método português no contexto considerado.
\end{abstract}

Palavras-chave: acessibilidade estrutural; transporte e uso do solo.

Abstract: The Structural Accessibility Layer (SAL) is the geographical representation of comparative accessibility levels by types of transport to different types of opportunities generating travel. The tool is based on integrated considerations regarding transport and land use systems. It was validated using the case study of its application on Greater Oporto. This paper aims at analyzing the accessibility levels of São Carlos using the proposed method. To accomplish this, Census data and results from an Origin and Destination survey were used. Thus, this study shows the Structural Accessibility Layer as a feature that supports the urban transportation planning, as can be observed in the application conducted in the city of São Carlos. In addition, the study supports the transferability of the application of SAL to the Brazilian case.

Keywords: structural accessibility; transportation and land use.

\section{INTRODUÇÃO}

O crescimento rápido e contínuo das cidades tem gerado problemas de mobilidade urbana em muitos lugares do mundo. Cada vez mais complexo, o funcionamento dos centros urbanos e dos seus sistemas de transportes exige soluções elaboradas para garantir a sua sustentabilidade. O presente trabalho vem ao encontro dessas necessidades, ao propor a utilização de uma ferramenta de suporte ao planejamento do transporte urbano. Baseada no conceito de Acessibilidade Estrutural, cuja proposta principal é avaliar como a estrutura urbana restringe as opções de viagem (Silva, 2008, 2013; Silva e Pinho, 2010), a ferramenta denominada Mapa de Acessibilidade Estrutural representa a distribuição geográfica dos níveis de acessibilidade considerando o uso de solo.

Ao aplicar o Mapa de Acessibilidade Estrutural desenvolvido por Silva (2008), se pretende validar duas hipóteses: a primeira diz que a cidade de São Carlos tem a sua rede de transporte desenhada de modo a privilegiar os modos de transporte individuais, a segunda considera que o método português é adequado para a implementação no contexto brasileiro. Assim, os objetivos do trabalho são: avaliar a acessibilidade estrutural na cidade de São Carlos, e comparar os resultados obtidos com os mostrados por Silva (2008), em seu estudo de caso na cidade do Porto, Portugal.

Uma revisão da bibliografia indica que o comportamento do usuário no sistema de transporte é influenciado

\footnotetext{
1 Denise Capasso da Silva, Departamento de Engenharia de Transportes, USP. (denise.capasso.silva@usp.br)

2 Antônio Nélson Rodrigues da Silva, Departamento de Engenharia de

Transportes, USP. (anelson@sc.usp.br)
}

Manuscrito recebido em 28/02/2016 e aprovado para publicação em $31 / 08 / 2016$

Este artigo é parte de TRANSPORTES v. 24, n. 3, 2016. ISSN: 2237-1346 (online). DOI:10.4237/transportes.v24i3.1082 pelo uso do solo (Ewing e Cervero, 2001; Wee, 2002; Wegener e Fürst, 1999). Sendo assim, a importância da integração do planejamento do sistema de transporte com a distribuição geográfica das atividades urbanas é amplamente reconhecida no meio acadêmico (Cervero, 2003; Halden, 2002; UN-Habitat, 2013). Nesse sentido, é válido o desenvolvimento de ferramentas que visam facilitar a ligação entre o diagnóstico da situação atual e o plano de mobilidade desenvolvido para o futuro, de modo que a acessibilidade potencial seja favorecida.

Uma das formas de caracterizar a conexão entre o uso de solo e as políticas de planejamento do transporte urbano é através de medidas de acessibilidade (Bertolini et al., 2005; Rodrigues da Silva, 1998; Raia Junior, 2000). A acessibilidade, por sua vez, pode ser definida em função de diversos fatores, como por exemplo: medidas de utilidade ou medidas que derivam do tipo de atividade que gera viagens. Para efeito de realização desse trabalho, a acessibilidade será tratada como a facilidade com que se pode alcançar determinada atividade a partir de um ponto, considerando uma área de abrangência circular, com centro no ponto de origem e raio de tamanho variável de acordo com o modo de transporte em análise. Dessa forma, não será medido o número de viagens efetivamente realizadas, mas sim as viagens que poderiam potencialmente ser geradas.

Nesse contexto, Silva (2008 e 2013) desenvolve em seus trabalhos um método de elaboração do Mapa de Acessibilidade Estrutural. Por ser o principal elemento conceitual do presente estudo, a revisão do material que documenta esse tema será feita em mais detalhes no item 2.

\section{MAPA DE ACESSIBILIDADE ESTRUTURAL}

O Mapa de Acessibilidade Estrutural, SAL (do termo em inglês - Structural Accessibility Layer) é, por definição, a representação geográfica comparativa dos níveis de acessibilidade, por modo de transporte, às diferentes oportunidades que geram viagens. 
Tabela 1. Instrumentos de acessibilidade em desenvolvimento na Europa Fonte: Papa et al., 2015

\begin{tabular}{|c|c|c|}
\hline Ano & Sigla & Método \\
\hline 2014 & ATRaPT & $\begin{array}{l}\text { Ferramenta de acessibilidade para análise do tempo de viagem rodoviária e no transporte público } \\
\text { (Accessibility tool for road and public transport travel time analysis) }\end{array}$ \\
\hline 2012 & ASAMeD & $\begin{array}{c}\text { Sintaxe Espacial: Integração espacial da acessibilidade e análise do segmento angular por distância } \\
\text { métrica (Space Syntax: Spatial integration accessibility and angular segment analysis by metric } \\
\text { distance) }\end{array}$ \\
\hline 2012 & ATI & $\begin{array}{c}\text { Da acessibilidade até o desenvolvimento do uso do solo (From accessibility to land development } \\
\text { potential) }\end{array}$ \\
\hline 2012 & EMM & $\begin{array}{c}\text { Atlas da acessibilidade para a região metropolitana de Munique (Erreichbarkeitsatlas der } \\
\text { Europäischen Metropolregion Muenchen) }\end{array}$ \\
\hline 2012 & GDATI & $\begin{array}{c}\text { Acessibilidade geográfica/demográfica da infraestrutura de transportes (Geographic/Demographic } \\
\text { Accessibility of Transport infrastructure) }\end{array}$ \\
\hline $2012 / 2013$ & GraBAM & $\begin{array}{l}\text { Acessibilidade baseada em medidas gravitacionais para uso na integração de transporte e uso do solo } \\
\text { (Gravity Based Accessibility Measures for Integrated Transport-Land Use Planning) }\end{array}$ \\
\hline 2013 & HIMMELI & $\begin{array}{c}\text { Instrumento heurístico de três níveis combinando morfologia urbana, mobilidade e ambientes de servi- } \\
\text { ços (Heuristic three-level Instrument Combining Urban Morphology, Mobility, and Service } \\
\text { Environments) }\end{array}$ \\
\hline 2012 & $\mathrm{IMaFa}$ & Mapas isocrônicos para benfeitorias (Isochrone Maps to Facilities) \\
\hline 2013 & INViTo & Ferramenta de visualização interativa (Interactive Visualization Tool) \\
\hline 2012 & JAD & Projeto de acessibilidade articulada (Joint-Accessibility Design) \\
\hline 2012 & MaReSi SC & $\begin{array}{c}\text { Método para alcançar o tamanho máximo recomendável para Shopping Centers (Method for Arriving } \\
\text { at Maximum Recommendable Size of Shopping Centres) }\end{array}$ \\
\hline 2010 & MARS & Simulador de realocação de atividades metropolitanas (Metropolitan Activity Relocation Simulator) \\
\hline 2012 & MoSC & $\begin{array}{c}\text { Medidas de conectividade em ruas: linhas espacialistas (Measures of Street Connectivity: Spatialist } \\
\text { Lines) }\end{array}$ \\
\hline 2005 & PST & Ferramenta de sintaxe local (Place Syntax Tool) \\
\hline 2012 & RIN & $\begin{array}{c}\text { Diretrizes alemãs para projetos e ligações de padrões de acessibilidade em redes integradas (German } \\
\text { Guidelines for Integrated Network Design-Binding Accessibility Standards) }\end{array}$ \\
\hline 2011 & SNAMUTS & $\begin{array}{c}\text { Análise de redes espaciais para sistemas de transportes multimodais (Spatial Network Analysis for } \\
\text { Multimodal Urban Transport Systems) }\end{array}$ \\
\hline 2012 & SNAPTA & $\begin{array}{c}\text { Análise espacial de redes para acessibilidade em transportes públicos (Spatial Network Analysis of Pu- } \\
\text { blic Transport Accessibility) }\end{array}$ \\
\hline 2012 & SoSINeTI & $\begin{array}{c}\text { Mudanças espaciais e sociais derivadas de novas infraestruturas de transportes (Social Spatial } \\
\text { Changes because of New Transport Infrastructure) }\end{array}$ \\
\hline 2012 & TRACE & Acessibilidade agrupada de varejo (Retail Cluster Accessibility) \\
\hline 2012 & UrbCA & $\begin{array}{c}\text { Modelo para apreciação da acessibilidade em planos espaciais (Cellular Automata Modelling for } \\
\text { Accessibility Appraisal in Spatial Plans) }\end{array}$ \\
\hline
\end{tabular}

Por si só ele não mede a mobilidade de uma região. Definido por Silva (2008) como uma ferramenta de suporte ao planejamento urbano, foi validado a partir do estudo de caso para a região metropolitana da cidade do Porto, Portugal. A ferramenta parte do princípio de que a acessibilidade pode ser usada para medir como o sistema de transporte interage com o uso do solo (Bertolini et al., 2005).

Nesse contexto, Papa et al. (2015) faz um levantamento das ferramentas de mensuração da acessibilidade integrada com o uso do solo em desenvolvimento na Europa e compara as medidas propostas. A Tabela 1 relaciona os instrumentos de acessibilidade estudados pelos autores e cujas principais finalidades foram agrupadas e colocadas no quadro comparativo mostrado na Figura 1. As mais recorrentes metas identificadas das ferramentas de medida da acessibilidade são quatro: decidir locação de atividades; gerenciar o uso de um modo de transporte em particular; garantir equidade econômica; garantir coesão e equidade social e estimular o desenvolvimento econômico. Dessa forma, três grandes grupos foram destacados de acordo com seus objetivos comuns: ferramentas de gerenciamento do uso do solo, ferramentas de planejamento de transportes e ferramentas multi-propósito. O método proposto neste artigo, Mapa de Acessibilidade Estrutural, SAL, segundo a sua própria autora, enquadra-se no grupo de ferramentas de planejamento de transportes, tendo como objetivo principal gerenciar o uso de um modo de transporte em particular.
A autora deixa espaço para adaptações no método para que o mesmo seja utilizado em outros contextos. São as chamadas escolhas de caso, que calibram o modelo para a aplicação específica em lugar distinto daquele em que o método foi concebido. Essa funcionalidade da ferramenta define um dos seus pontos fortes: a adaptabilidade a cenários diversos, permitindo que a mesma tenha potencial para ser aplicada em lugares distintos. Sendo assim, devem ser adaptadas, para cada aplicação: fronteiras da região de estudo; nível de desagregação espacial; frequência potencial de uso $\left(f_{y}\right.$ - coeficiente utilizado como fator de ponderação da importância do acesso à atividade $y$ ); nível de desagregação por atividade e critérios de corte e valores (banda de influência de cada região de estudo para cada um dos modos de transporte - fronteira de acessibilidade).

Depois de definidas as condições específicas do caso é calculada, pela Equação (1), a variável DivAct - Diversidade de atividades potencialmente atingíveis. Ela representa a diversidade de atividades que pode ser acessada a partir do centro de uma região de estudo (definida pelo nível de desagregação espacial). Ela é função da variável $A c t_{y}$, que indica a presença $\left(A c t_{y}=1\right)$ ou não $\left(A c t_{y}=0\right)$ da atividade $y$ na fronteira de acessibilidade considerada (função dos critérios de corte definidos). 


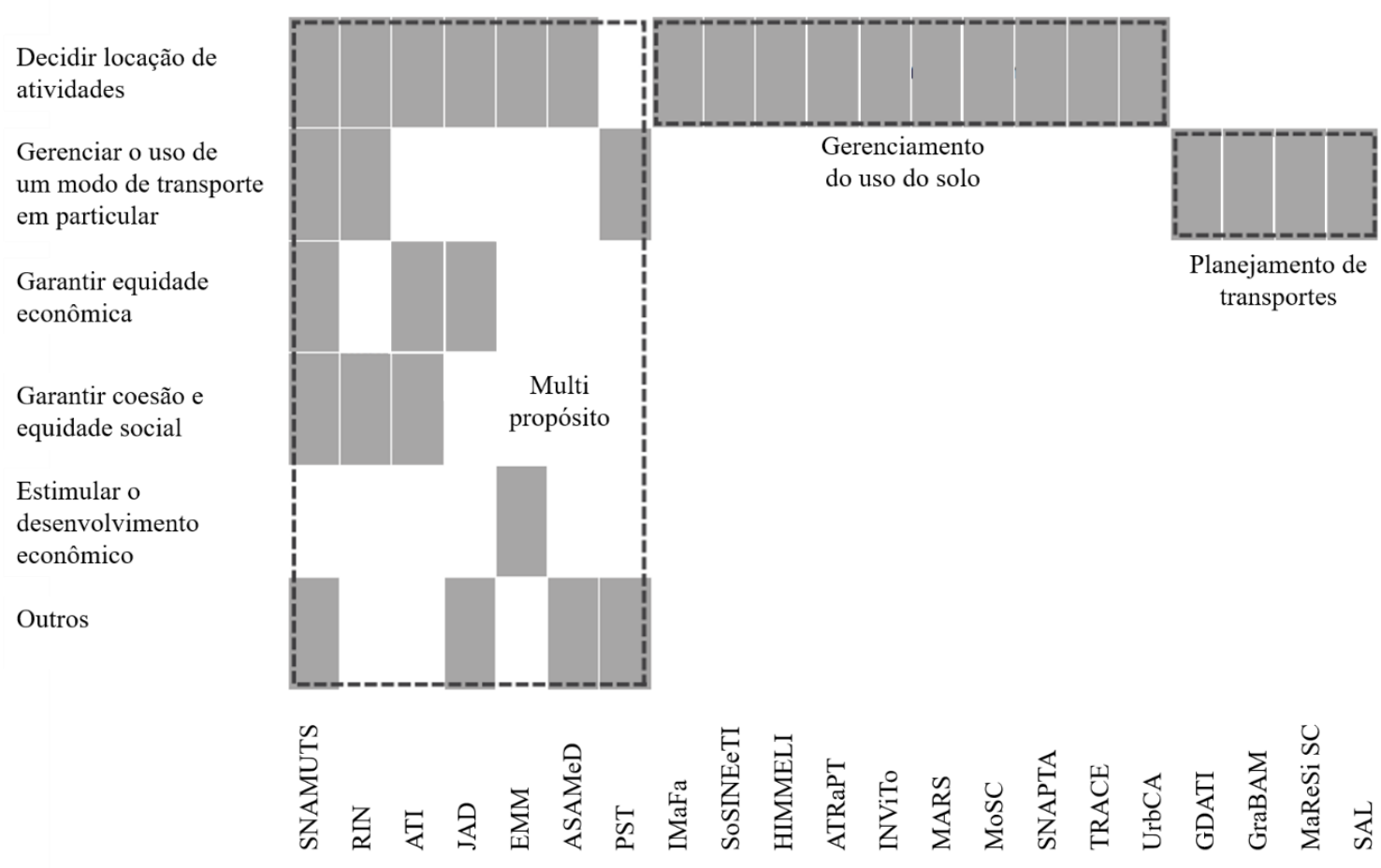

Figura 1. Quadro comparativo dos instrumentos de acessibilidade em desenvolvimento na Europa Fonte: Papa et al., 2015

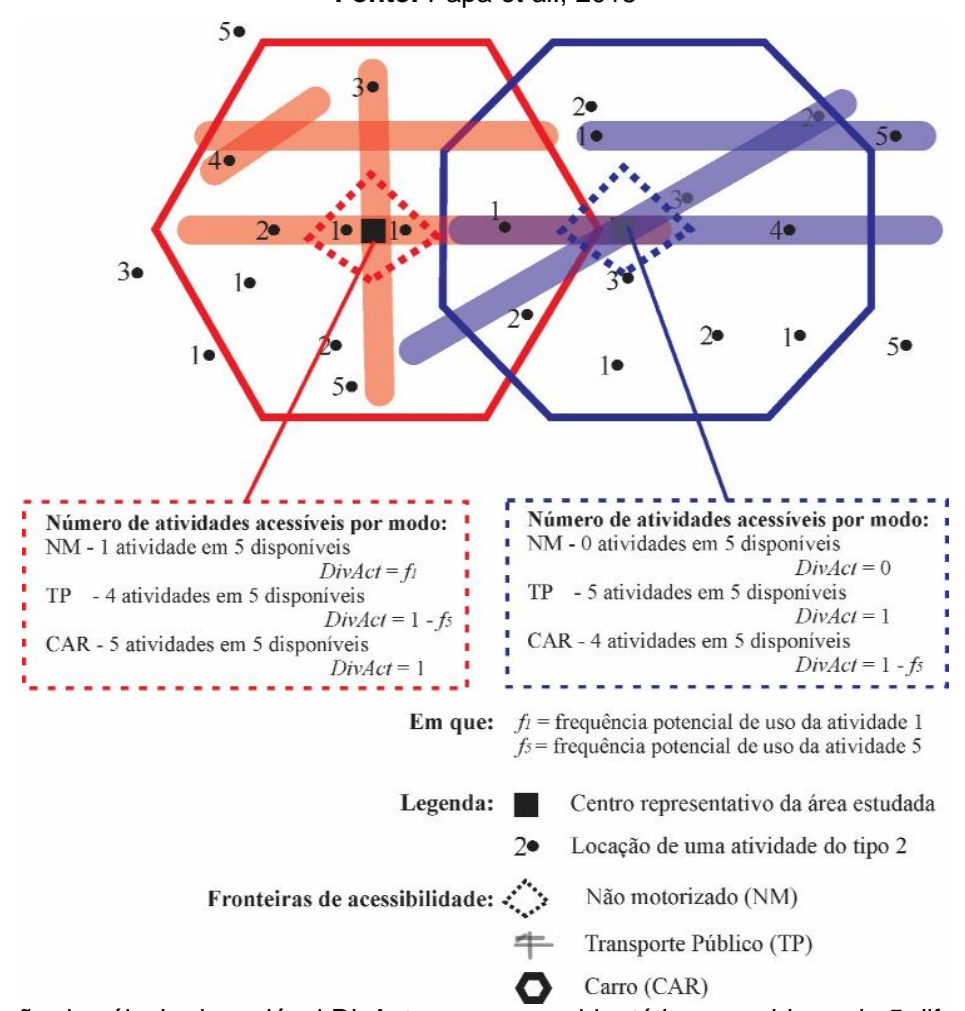

Figura 2. llustração de cálculo da variável DivAct em um caso hipotético, considerando 5 diferentes atividades Fonte: Adaptado de Silva, 2008

No caso da atividade relacionada às viagens por motivo de trabalho, considera-se que, quando o número de viagens a trabalho atraídas por setor censitário supera a metade no número de habitantes que lá residem, a atividade "Emprego" está presente; caso contrário, considera-se que a atividade está ausente. A variável DivAct - Diversidade de atividades potencialmente atingíveis também é função de $f_{y}$, que representa a frequência potencial de uso definida de acordo com a periodicidade em que a atividade é realizada e a distribuição de viagens por modo.

$$
\operatorname{DivAct}=\frac{\sum_{y}\left(A c t_{y} * f_{y}\right)}{\sum_{y} f_{y}}
$$

Sendo assim, DivAct varia no intervalo de 0 a 1 , em que o valor nulo representa a situação em que nenhuma atividade pode ser atingida quando se considera a fronteira de acessibilidade em questão, e o valor unitário representa a situação oposta, em que todas as atividades podem ser acessadas. Na Figura 2 encontra-se ilustrada a determinação da 
variável DivAct para um caso hipotético, simplificado com apenas duas áreas de estudo e cinco atividades.

Uma vez calculados os valores da variável DivAct para os modos de transporte considerados, se define classes de acessibilidade para cada um dos modos de transporte considerados. Os intervalos propostos por Silva (2008) estão especificados na Tabela 2.

Tabela 2. Intervalos da variável DivAct - Diversidade de atividades potencialmente atingíveis por classe de acessibilidade definida Fonte: Silva, 2008

\begin{tabular}{lc}
\hline Classe & Intervalo \\
\hline Classe A (alto nível de acessibilidade) & {$[0,85 ; 1,00]$} \\
Classe B (médio nível de acessibilidade) & {$[0,50 ; 0,85[$} \\
Classe C (baixo nível de acessibilidade) & {$[0,00 ; 0,50[$} \\
\hline
\end{tabular}

A variável que representa a sustentabilidade no modelo é definida de maneira gráfica, como exemplificado na Figura 3. Os valores encontrados para a DivAct - Diversidade de atividades potencialmente atingíveis para cada um dos três modos de transporte escolhidos (não motorizado, transporte público e automóvel) são dispostos em um sistema de coordenadas tridimensionais. $\mathrm{O}$ resultado pertence a um cubo de lado 1, representando as possíveis combinações dos valores encontrados.

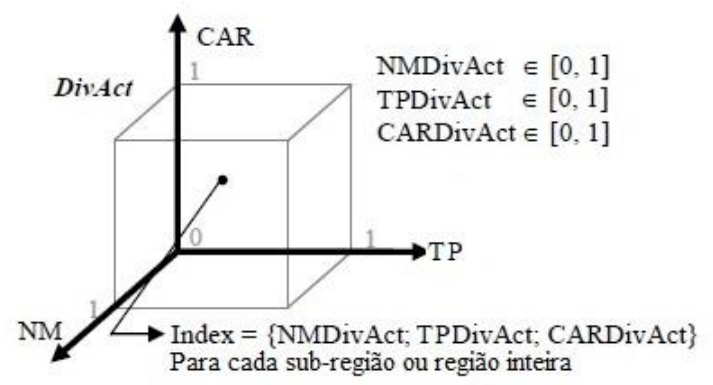

Para a criação do Mapa de Acessibilidade Estrutural, cada um dos eixos é dividido de acordo com as classes propostas na Tabela 2. Dessa forma, o cubo de comparação fica definido em 27 categorias de acessibilidade que representam as possíveis combinações de resultados para a variável DivAct - Diversidade de atividades potencialmente atingíveis. Silva (2008) então define grupos de acessibilidade de acordo com as categorias possíveis. A representação gráfica da definição dos grupos de acessibilidade pode ser encontrada na Figura 4.

Para ilustrar o método empregado, a Figura 5 apresenta um fluxograma que detalha as suas etapas de forma a possibilitar uma melhor compreensão dos procedimentos de cálculo.

\section{METODOLOGIA}

Para a realização do primeiro objetivo deste trabalho, que é a análise da acessibilidade estrutural através do desenvolvimento de um Mapa de Acessibilidade Estrutural para a cidade de São Carlos, foi utilizado o método proposto por Silva (2008). Segundo a autora, algumas definições devem ser feitas ao implementar o método em outra localidade. Assim, foram definidas as escolhas específicas do caso, para calibrar o modelo à situação em que ele é aplicado. São elas: definição de fronteiras da região de estudo,

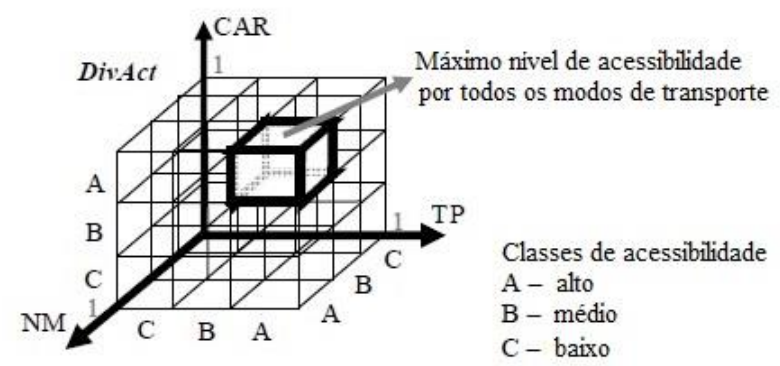

Figura 3. Definição gráfica da variável comparativa de acessibilidade, considerando os modos de transporte NM - Não motorizado, TP Transporte Público e CAR - Carro

Fonte: Adaptado de Silva, 2008

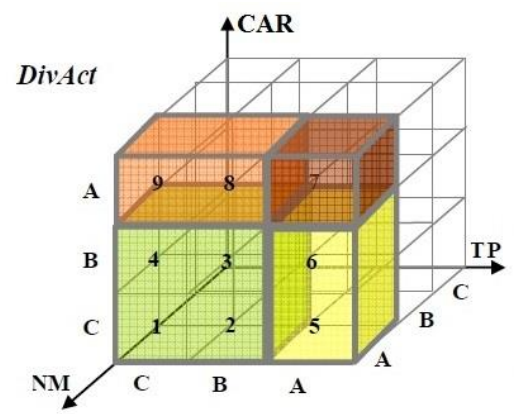

Classes de acessibilidade: A- alto; B- médio; C- baixo.

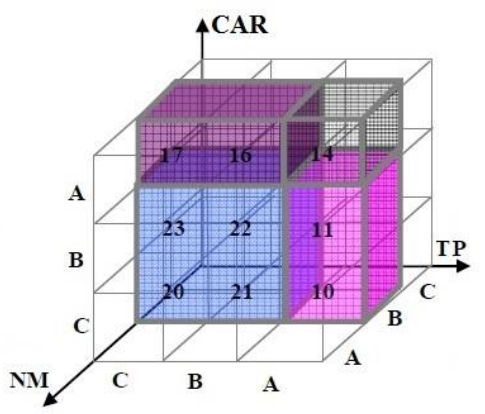

Categoria de acessibilidade: $\square$ a 27

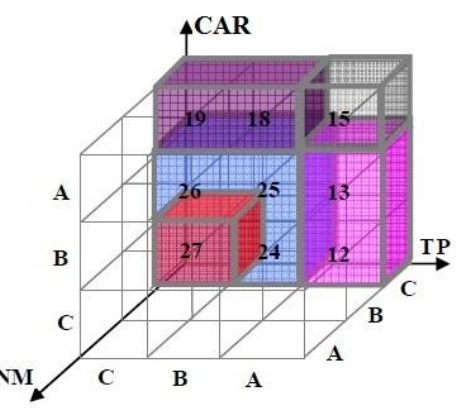

\footnotetext{
Grupos

I - Condições favoráveis para NM (1 a 4)

II - Condições favoráveis para NM e TP (5 e 6)

III - Condições favoráveis para todos os modos (7)

IV - Condições favoráveis para NM e CAR (8 e 9)

V - Condições favoráveis para TP (10 a 13)
}

Figura 4. Definição dos grupos de acessibilidade, considerando os modos de transporte NM - Não motorizado, TP Transporte Público e CAR - Carro

Fonte: Adaptado de Silva, 2008 


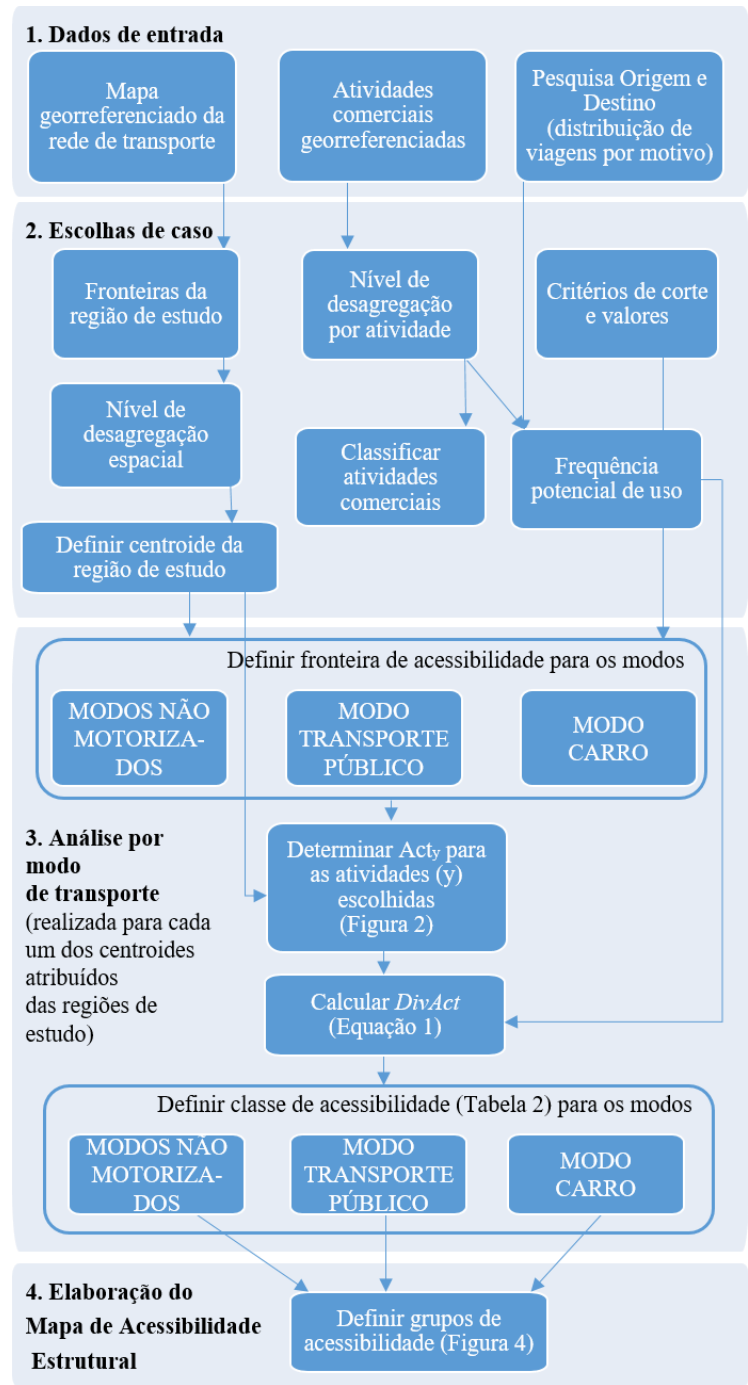

Figura 5. Ilustração do método de desenvolvimento do Mapa de Acessibilidade Estrutural

nível de desagregação espacial, nível de desagregação por atividade, frequência potencial de uso $\left(f_{y}\right)$ e critérios de corte e valores.

Como definição de fronteira da região de estudo e nível de desagregação espacial, no Brasil pode-se utilizar como unidade de área para estudo os setores censitários propostos pelo Instituto Brasileiro de Geografia e Estatística (IBGE, 2015).

As atividades consideradas, representando o nível de desagregação por atividade, foram identificadas a partir do catálogo de endereços do IBGE, e categorizadas de acordo com a proposição feita por Silva (2008). Sendo assim, considerou-se:

1. Educação Infantil: escolas de educação infantil, ensino fundamental (I e II), creches e berçários;

2. Ensino Médio: instituições de ensino médio;

3. Ensino Superior: instituições de ensino superior faculdades e universidades;

4. Atividades Complementares: escolas de idiomas, centros de aprofundamento de aprendizado, aulas de reforço e professores particulares;

5. Restaurantes: lanchonetes, restaurantes, pizzarias, bem como bares e boates que servem refeições;
6. Esportes: ginásios, clubes, academias, centros de dança e demais espaços cuja finalidade é a prática esportiva;

7. Lazer - outros: demais atividades de cultura e lazer, como bibliotecas e cinemas, entre outros;

8. Comida: comércio de produtos alimentícios, como supermercados, quitandas, mercearias e sacolões de verduras;

9. Serviços: barbeiros, alfaiates, salões de beleza, escritórios virtuais, saúde animal, serviços elétricos e automotivos, entre outros;

10. Comércio: comércio de bens, novos e usados, à exceção de produtos alimentícios;

11. Igrejas: igrejas, templos, centros de reunião com fins espirituais e religiosos;

12. Farmácias: comércios de medicamentos e produtos hospitalares;

13. Hospitais e Clínicas: instituições de saúde, como hospitais, unidades de saúde da família, clínicas médicas e odontológicas, bem como centros de exames e diagnósticos;

14. Departamentos Municipais: órgãos que servem o governo municipal, como Prefeitura, Câmara dos Vereadores e demais Secretarias;

15. Correios: agências prestadoras de serviços postais;

16. Bancos: agências bancárias; 
Tabela 3. Níveis de desagregação por atividade e frequência potencial de uso $\left(f_{y}\right)$ adotada na cidade de São Carlos

\begin{tabular}{|c|c|c|c|}
\hline Atividades (Adaptado de Silva, 2008) & Periodicidade & $\begin{array}{c}\text { \% viagens } \\
\text { (Rodrigues da Silva, 2008) }\end{array}$ & $f_{y}$ \\
\hline \multicolumn{4}{|l|}{ Educação } \\
\hline Educação Infantil & Diária & \multirow{4}{*}{31} & 9 \\
\hline Ensino Médio & Diária & & 9 \\
\hline Ensino Superior & Diária & & 9 \\
\hline Atividade Complementar & Semanal & & 4 \\
\hline \multicolumn{4}{|l|}{ Lazer } \\
\hline Parques e praças & Esporádico & \multirow{4}{*}{5} & 1 \\
\hline Restaurante & Semanal & & 2 \\
\hline Esporte & Semanal & & 1 \\
\hline Outros = Lazer & Esporádico & & 1 \\
\hline \multicolumn{4}{|l|}{ Assuntos Pessoais } \\
\hline Comida & Semanal & \multirow{4}{*}{8} & 4 \\
\hline Serviços & Mensal & & 1 \\
\hline Comércio & Mensal & & 1 \\
\hline Igrejas & Semanal & & 2 \\
\hline \multicolumn{4}{|l|}{ Saúde } \\
\hline Farmácias & Esporádico & \multirow{2}{*}{4} & 2 \\
\hline Hospitais e Clínicas & Esporádico & & 2 \\
\hline \multicolumn{4}{|l|}{ Outros Assuntos } \\
\hline Departamentos Municipais & Esporádico & \multirow{4}{*}{14} & 3 \\
\hline Correios & Esporádico & & 3 \\
\hline Bancos & Mensal & & 5 \\
\hline Outros (seguros, advogados, arquitetos, etc) & Esporádico & & 3 \\
\hline \multicolumn{4}{|l|}{ Trabalho } \\
\hline Empregos & Diária & 38 & 38 \\
\hline \multicolumn{2}{|l|}{ Total } & 100 & 100 \\
\hline
\end{tabular}

17. Outros: demais atividades, incluindo profissionais liberais vinculados às atividades de advocacia, engenharia, arquitetura, contabilidade, seguros, previdências e afins;

18. Empregos.

Silva (2008) define que há presença da atividade "Emprego" $\left(A c t_{y}=1\right)$ quando o número de empregos em determinada área excede, em números absolutos, metade da população que lá habita.

De maneira análoga, quando a relação Emprego/ População é inferior a 0,5 ou $50 \%$, considera-se $A c t_{y}=0$. Para adaptar essa definição aos dados disponíveis para a cidade de São Carlos utilizou-se como base os resultados fornecidos por Tan (2013), onde o número de viagens por motivos de trabalho atraídas em cada uma das zonas de tráfego da cidade de São Carlos foi estimado com base no relatório da pesquisa de origem e destino realizada em 2007-2008 (Rodrigues da Silva, 2008). Considerando que o número de viagens atraídas por motivo de trabalho corresponde numericamente ao número de empregos existentes no setor censitário, foi possível estabelecer a relação proposta. Determinou-se assim, a partir da taxa de viagens atraídas por trabalho, a relação Emprego/População de cada uma das áreas de estudo. Não foram considerados: galpões, depósitos, pontos comerciais vagos, estacionamentos, garagens, estabelecimentos industriais, atividades de agricultura, pecuária, apicultura.

A frequência potencial de uso $\left(f_{y}\right)$ pode ser admitida a partir da distribuição das viagens por motivo. No caso de São Carlos, essa informação pôde ser obtida a partir da pesquisa de origem e destino realizada em 2007-2008 (Rodrigues da Silva, 2008), cujos resultados estão representados de forma resumida na Figura 6. A lista das atividades com suas respectivas frequências potenciais de uso está mostrada na Tabela 3.
Os critérios de corte foram utilizados para a definição das fronteiras de acessibilidade por modo de transporte. A adoção dos valores para as bandas da fronteira de acessibilidade foi feita de acordo com a sensibilidade de sua definição no resultado. Assim, adotou-se para o estudo: 750 metros para modos não motorizados, dois quilômetros para o transporte público e três quilômetros para o modo carro. As bandas definidas no critério de corte foram implementadas a partir do centroide de cada área de estudo, com raio variando de acordo com o modo de transporte utilizado. Para o caso do transporte público, admitiu-se que estão acessíveis pela rede apenas as atividades que se encontram a, no máximo, 250 metros de qualquer ponto de parada da linha de ônibus.

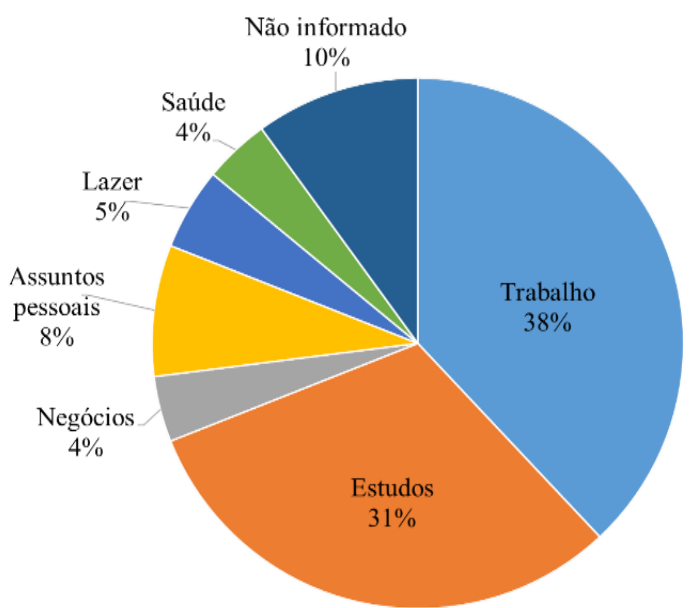

Figura 6. Motivos de viagens informados em Pesquisa OrigemDestino realizada na cidade de São Carlos

Fonte: Rodrigues da Silva, 2008

No caso dos setores censitários de maior área que se encontravam muito afastados do centro comercial da cidade, foi necessário um ajuste na consideração do centroide 


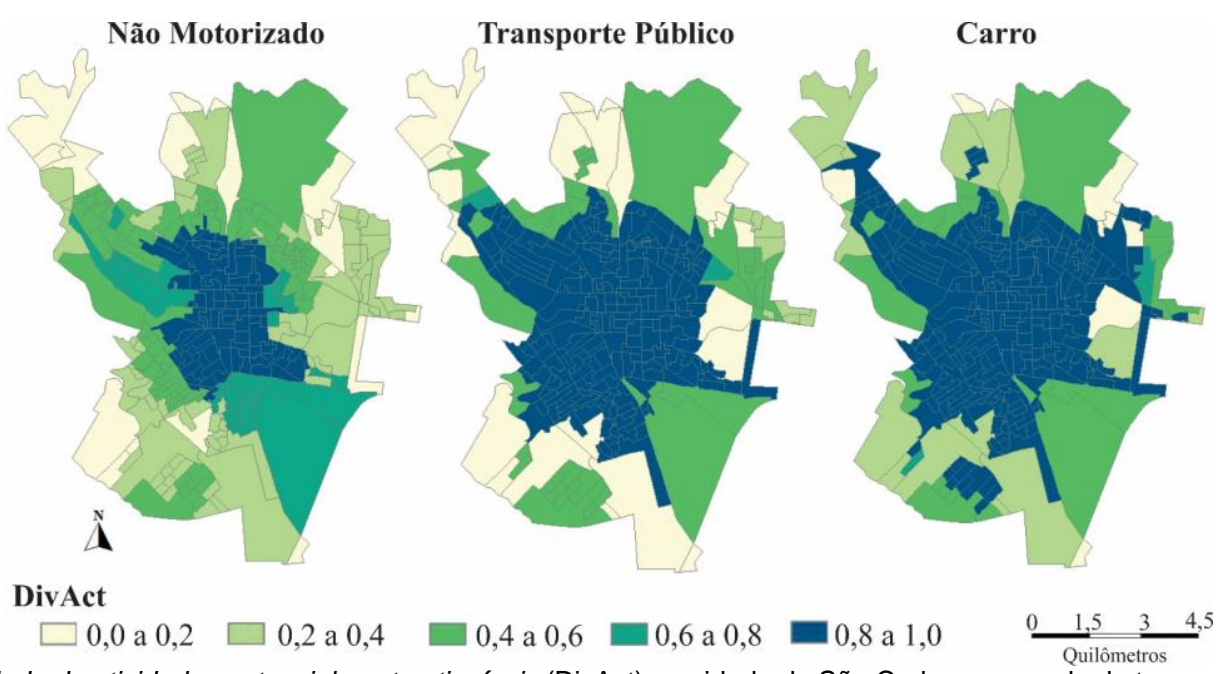

Figura 7. Diversidade de atividades potencialmente atingíveis (DivAct) na cidade de São Carlos, por modo de transporte, onde 0 representa nenhuma atividade acessível e 1 representa todas as atividades acessíveis

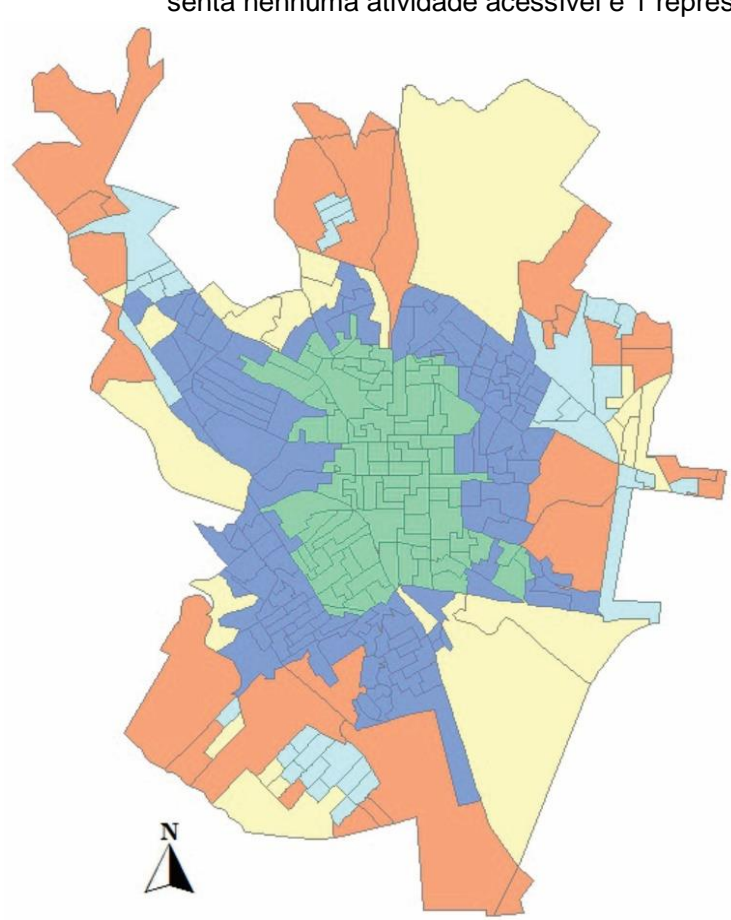

Grupos

Figura 8. Mapa de Acessibilidade Estrutural da cidade de São Carlos

da região de estudo para o cálculo da banda. A fim de melhor representar a distribuição da população inserida nessas regiões, o ponto de partida das fronteiras de acessibilidade foi deslocado para regiões do setor em que a taxa de ocupação é maior, em geral mais próximas do centro da cidade.

Definidos os parâmetros de adaptação do método, a variável que representa a Diversidade de atividades potencialmente atingíveis (DivAct) foi calculada para todos os setores censitários da cidade, considerando os modos não motorizados, transporte público e automóvel. Primeiramente essa variável foi representada em mapas com cinco classes, onde se observa a transição dos níveis de acessibilidade por modo de transporte. Posteriormente, foi montado o Mapa de Acessibilidade Estrutural representando os grupos de acessibilidade encontrados.

Para análise dos resultados, foi realizada comparação com aplicação disponível na literatura. Tendo em vista que, até o momento do desenvolvimento do trabalho, apenas dois estudos de caso haviam sido publicados, o primeiro na cidade do Porto (Silva, 2008) e o segundo na cidade dinamarquesa de Copenhague (Reis, 2009), a cidade portuguesa foi escolhida como referência. Entende-se que são contextos urbanos bastante distantes, e inclusive de diferentes dimensões (São Carlos possui cerca de 200 mil habitantes, enquanto a região metropolitana do Porto abrange mais de 1 milhão de pessoas), porém, devido à ausência de recursos melhores, admitiu-se que as aplicações eram semelhantes o suficiente para uma análise comparativa. Dessa forma, uma comparação dos resultados obtidos em São Carlos e na cidade do Porto foi realizada através da variável DivAct, de acordo com a segmentação proposta por Silva (2008) em classes de acessibilidade A (alto, DivAct $\geq 0,85$ ), B (médio, $0,50 \leq D i v A c t<0,85$ ) e C (baixo, DivAct $<0,50$ ), com suas respectivas influências em área e população. Também foram comparadas nas duas cidades as distribuições dos grupos de acessibilidades encontrados de acordo com sua área e população.

\section{RESULTADOS}

Aplicando o método proposto por Silva (2008) nos dados obtidos para a cidade de São Carlos, foi possível 
Tabela 4. Distribuição comparativa por população das classes de acessibilidade de acordo com a Diversidade de atividades potencialmente atingíveis (DivAct) nas cidades de São Carlos e do Porto Fonte: Adaptado de Silva, 2008

\begin{tabular}{ccccccc}
\hline & \multicolumn{3}{c}{ São Carlos (210 mil hab.) } & \multicolumn{2}{c}{ Porto (1 milhão hab.) } \\
\cline { 2 - 6 } & NM & TP & CAR & NM & TP & CAR \\
\hline DivAct $=1,00$ & $7,4 \%$ & $62,2 \%$ & $75,6 \%$ & $25,2 \%$ & $71,2 \%$ & $98,1 \%$ \\
A $(0,85 \leq$ DivAct $\leq 1,00)$ & $20,3 \%$ & $65,0 \%$ & $78,8 \%$ & $77,6 \%$ & $83,4 \%$ & $100 \%$ \\
B $(0,50 \leq$ DivAct $<0,85)$ & $18,9 \%$ & $11,0 \%$ & $8,6 \%$ & $21,3 \%$ & $1,7 \%$ & - \\
C (DivAct $<0,50)$ & $60,8 \%$ & $24,0 \%$ & $12,6 \%$ & $1,1 \%$ & $15,0 \%$ & - \\
DivAct $=0,00$ & $0,1 \%$ & $8,6 \%$ & $2,7 \%$ & - & $15,0 \%$ & - \\
\hline
\end{tabular}

Tabela 5. Distribuição comparativa por área das classes de acessibilidade de acordo com a Diversidade de atividades potencialmente atingíveis (DivAct) nas cidades de São Carlos e do Porto Fonte: Adaptado de Silva, 2008

\begin{tabular}{ccccccc}
\hline & \multicolumn{3}{c}{ São Carlos $\left(\mathbf{8 0} \mathbf{~ k m}^{2}\right)$} & \multicolumn{3}{c}{ Porto $\left(\mathbf{5 6 3} \mathbf{~ k m}^{2}\right)$} \\
\cline { 2 - 6 } & NM & TP & CAR & NM & TP & CAR \\
\hline DivAct $=1,00$ & $5,3 \%$ & $33,1 \%$ & $41,7 \%$ & $7,3 \%$ & $31,3 \%$ & $86,5 \%$ \\
A $(0,85 \leq$ DivAct $\leq 1,00)$ & $13,3 \%$ & $35,5 \%$ & $43,5 \%$ & $43,5 \%$ & $47,8 \%$ & $100 \%$ \\
B (0,50 $\leq$ DivAct $<0,85)$ & $18,8 \%$ & $30,1 \%$ & $27,0 \%$ & $48,6 \%$ & $4,0 \%$ & - \\
C (DivAct $<0,50)$ & $67,9 \%$ & $34,5 \%$ & $29,5 \%$ & $7,9 \%$ & $48,2 \%$ & - \\
DivAct $=0,00$ & $0,5 \%$ & $27,9 \%$ & $5,2 \%$ & - & $48,2 \%$ & - \\
\hline
\end{tabular}

Tabela 6. Distribuição comparativa dos grupos de acessibilidade por área e população nas cidades de São Carlos e do Porto Fonte: Adaptado de Silva, 2008

\begin{tabular}{lcccc}
\hline \multirow{2}{*}{ Grupo de acessibilidade } & \multicolumn{2}{c}{ São Carlos } & \multicolumn{2}{c}{ Porto } \\
\cline { 2 - 5 } & Área & População & Área & População \\
\hline III Condições favoráveis para todos os modos & $13,3 \%$ & $20,3 \%$ & $32,1 \%$ & $70,7 \%$ \\
IV Condições favoráveis para NM E CAR & - & - & $11,3 \%$ & $6,8 \%$ \\
VI Condições favoráveis para TP e CAR & $22,2 \%$ & $12,6 \%$ & $15,5 \%$ & $12,7 \%$ \\
VII Condições favoráveis para CAR & $8,0 \%$ & $44,8 \%$ & $41,0 \%$ & $9,8 \%$ \\
VIII Níveis médios de acessibilidade & $27,0 \%$ & $13,8 \%$ & - & - \\
IX Baixos níveis de acessibilidade & $29,5 \%$ & $8,6 \%$ & - & - \\
\hline
\end{tabular}

construir o Mapa de Acessibilidade Estrutural. A primeira etapa deste processo foi a definição dos valores da diversidade de atividades para cada meio de transporte, através da variável DivAct. A distribuição desses índices para os modos de transportes analisados está representada na Figura 7.

Assim, a leitura da Figura 7, de acordo com a população que habita os setores censitários da cidade, permite, por exemplo, a seguinte interpretação: considerando os modos não motorizados, $21,2 \%$ das pessoas vivem em regiões cuja diversidade de atividades potencialmente atingíveis está entre 0,8 e 1,0, ou seja, permite atingir $80,0 \%$ ou mais das atividades consideradas. Ao analisar o modo carro, a parcela de população que possui aproximadamente o mesmo nível de acessibilidade sobe para 78,8\% (Tabela 4).

A partir dos valores de diversidade de atividades ( $D i$ $v A c t$ ), atribuídos para cada um dos modos de transporte, foi elaborado o Mapa de Acessibilidade Estrutural, que está representado na Figura 8. Ele é uma ferramenta que pode ser utilizada no planejamento do transporte urbano, uma vez que evidencia as áreas da cidade em que os níveis de acessibilidade requerem maior atenção.

Também é possível comparar o resultado mostrado na Figura 8 com os dados de distribuição populacional por setor censitário, resultando em uma análise interessante de acessibilidade. Assim, o Mapa de Acessibilidade Estrutural mostra que $8,6 \%$ da população vivem em regiões da cidade em que são observados baixos níveis de acessibilidade (grupo IX), enquanto apenas 20,3\% dos habitantes residem em regiões com condições de acessibilidade favorável por todos os modos.

Com os resultados fornecidos pelo método proposto para a cidade de São Carlos, é possível a comparação com o estudo de caso feito por Silva (2008) na cidade portuguesa do Porto. A primeira análise possível é a da distribuição das classes de acessibilidade definidas, tanto em frações da população (Tabela 4), como em função da área referente a cada nível de acessibilidade (Tabela 5). Dessa forma, se tem que, enquanto em São Carlos apenas $20,3 \%$ da população vivem em regiões de acessibilidade classe A por modos não motorizados, na cidade do Porto esse número é de $77,6 \%$. Também é notável que, enquanto no caso europeu $100 \%$ da área analisada correspondia à uma região de acessibilidade classe A por automóvel, no caso são-carlense a área correspondente é de apenas $43,5 \%$.

Ao comparar a distribuição dos grupos de acessibilidade nas cidades em questão (Tabela 6), observa-se a distinção de mobilidade potencial oferecida nas duas localidades. Enquanto no caso europeu não há ocorrências de níveis baixos e médios de acessibilidades, no caso brasileiro não ocorrem condições favoráveis exclusivamente para modos não motorizados e por carro. Além disso, observa-se que, na cidade de São Carlos, 44,8\% da população são servidos com condições favoráveis de acessibilidade exclusivamente pelo modo automóvel, enquanto na cidade do Porto essa parcela é de apenas $9,8 \%$ dos habitantes.

\section{CONCLUSÕES}

A análise dos resultados expostos pode ser usada para confirmar a hipótese inicial do presente trabalho, em que se diz que a cidade de São Carlos tem a sua rede de transporte desenhada de modo a privilegiar o automóvel. O Mapa de Acessibilidade Estrutural obtido mostra que somente a região central da cidade possui bons níveis de acesso às atividades propostas por todos os modos de transportes. Quanto mais afastado desse centro comercial, que é ocupado de forma bastante variada, menores os índices de qualidade do 
acesso pelos modos não motorizados e transporte público. Essa condição implica em notável dependência dos modos motorizados individuais para grande parte da população.

Ao confirmar a hipótese apresentada se conclui também que a ferramenta retrata de forma adequada a realidade do transporte observada no município. Sendo assim, a ferramenta de análise da acessibilidade estrutural, feitas as devidas adaptações ao caso São-Carlense, se mostrou eficiente na identificação de áreas que exigem atenção da entidade planejadora de transportes.

Além das considerações a respeito do caso de estudo, se tem que a utilização do método de análise proposto em Portugal foi válida para a aplicação no Brasil, visto que os resultados se mostraram coerentes com a situação vivenciada em São Carlos. Dessa forma, se pode dizer também que as escolhas de caso feitas para a aplicação apresentada foram adequadas, já que conseguiu produzir resultado relevante e satisfatório mesmo em condições tão distintas da originalmente proposta. Ainda, ao contribuir para o melhor planejamento urbano do transporte, considerando a integração do mesmo com o uso e ocupação do solo, o Mapa de Acessibilidade Estrutural da cidade de São Carlos atende aos objetivos primeiros da criação da ferramenta por Silva (2008), validando a hipótese de que o método português é adequado para a implementação no cenário proposto. No entanto, considerando que o Brasil é um país de grandes contrastes internos, seria interessante a análise da aplicação do método em outras cidades, inclusive de outras regiões.

Indo além, o estudo realizado na cidade de São Carlos corrobora as conclusões do estudo que deu origem ao método aplicado, o Mapa de Acessibilidade Estrutural. Sendo assim, é possível dizer também que o SAL é uma ferramenta de diagnóstico adequada para análise e planejamento de sistemas de transporte, sendo um instrumento com notável capacidade sintetizadora de informações, e útil na visualização das restrições de mobilidade em uma cidade.

\section{AGRADECIMENTOS}

Os autores agradecem o financiamento da pesquisa pelo Conselho Nacional de Desenvolvimento Científico e Tecnológico (CNPq).

\section{REFERÊNCIAS}

Bertolini, L.; F. Clercq e L. Kapoen (2005) Sustainable Accessibility: a Conceptual Framework to Integrate Transport and Land Use Plan-Making. Two Test-Applications in the Netherlands and a Reflection on the Way Forward. Transport Policy, v. 12, n. 3, p. 207-220. DOI:

10.1016/j.tranpol.2005.01.006.

Cervero, R. (2003) Growing Smart by Linking Transportation and Land Use: Perspectives from California. Built Environment, v. 29 , n. 1, p. 66-78. DOI: 10.2148/benv.29.1.66.53948.

Ewing, R. e R. Cervero (2001) Travel and the Built Environment: a Synthesis. Transportation Research Record, v. 1780, pp. 89-91. DOI: $10.3141 / 1780-10$.

Halden. D. (2002) Using Accessibility Measures to Integrate Land Use and Transport Policy in Edinburgh and the Lothians. Transport Policy, v. 9 , n. 4, p. 313-324. DOI: 10.1016/S0967070X(02)00017-3.
IBGE (2015) Instituto Brasileiro de Geografia e Estatística. Acessado em 2015-01-30, de http://www.ibge.gov.br.

Papa, E.; C. Silva; M. Brömmelstroet e A. Hull (2015), Accessibility instruments for planning practice: A review of European experiences. The Journal of Transport and Land Use, v. 9 , n. 3, p. 1-20. DOI: 10.5198/jtlu.2015.585.

Raia Junior, A. A. (2000) Acessibilidade e Mobilidade na Estimativa de um Índice de Potencial de Viagens Utilizando Redes Neurais Artificiais e Sistemas de Informações Geográficas. Tese de Doutorado, Escola de Engenharia de São Carlos, Universidade de São Paulo, São Carlos. DOI: 10.11606/T.18.2000.tde-10112001-160812.

Reis, J. P. F. F. (2009) Urban Structure and Mobility - A case study in Copenhagen. Dissertação de Mestrado, Centro de Pesquisa Transporte e Ambiente, Universidade do Porto. Acessado em 2016-08-24, de http://hdl.handle.net/10216/58033.

Rodrigues da Silva, A. N. (1998) Sistemas de Informações Geográficas para Planejamento de Transportes. Tese de Livre Docência, Escola de Engenharia de São Carlos, Universidade de São Paulo, São Carlos. Acessado em 2015-07-15, de http://www.teses.usp.br/teses/disponiveis/livredocencia/18/tde03022006-154920/.

Rodrigues da Silva, A. N. (2008) Pesquisa Origem-Destino da cidade de São Carlos. Relatório do projeto de pesquisa FAPESP (Fundação de Amparo à Pesquisa do Estado de São Paulo) número 04/15843-4. São Carlos.

Silva, C. (2008) Comparative Accessibility for Mobility Management: The Structural Accessibility Layer. Tese de Doutorado, Centro de Pesquisa Transporte e Ambiente, Universidade do Porto. Acessado em 2016-08-24, de http://citta.fe.up.pt/publications/phd-theses/comparativeaccessibility-for-mobility-management-the-structuralaccessibility-layer.

Silva, C. (2013) Structural Accessibility for Mobility Management. Progress in Planning, v. 81, p. 1-49. DOI: 10.1016/j.progress.2012.07.001.

Silva, C. e P. Pinho (2010) The Structural Accessibility Layer (SAL): Revealing How Urban Structure Constrains Travel Choice. Environment and Planning A, v. 42, n. 11, p. 2735-2752. DOI: $10.1068 / \mathrm{a} 42477$.

Tan, F. (2013) Estudo das características de infraestrutura, tecnologia e operação de veículos leves sobre trilhos (VLT) visando à implantação em cidades de médio porte. Relatório de Iniciação Científica, Escola de Engenharia de São Carlos.

UN-Habitat (2013) Planning and Design for Sustainable Urban Mobility: Policy Directions. United Nations Human Settlements Programme, Nairobi; Earthscan from Routledge, Abingdon [etc.]. Acessado em 2016-08-24, de http://unhabitat.org/books/planning-and-design-for-sustainableurban-mobility-global-report-on-human-settlements-2013/.

Wee, B. (2002) Land Use and Transport: Research and Policy Challenges. Journal of Transport Geography v. 10, n. 4, p. 259271. DOI: 10.1016/S0966-6923(02)00041-8.

Wegener, J. e F. Fürst (1999) Land-Use Transport Interaction: State of the Art. TRANSLAND European Commission. DOI: $10.2139 /$ ssrn. 1434678 . 